Musées, Patrimoine et Culture scientifiques et techniques

$133 \mid 2011$

janvier - février 2011

\title{
Le développement durable dans la construction d'un Centre de Conservation et d'Étude
}

Sustainable development in the building of a Conservation and Study Centre

\section{Séverine Aubert}

\section{(2)enEdition}

Journals

Édition électronique

URL : http://journals.openedition.org/ocim/655

DOI : $10.4000 /$ ocim. 655

ISSN : 2108-646X

Éditeur

OCIM

Édition imprimée

Date de publication : 1 janvier 2011

Pagination : 18-23

ISSN : 0994-1908

\section{Référence électronique}

Séverine Aubert, «Le développement durable dans la construction d'un Centre de Conservation et d'Étude », La Lettre de I'OCIM [En ligne], 133 | 2011, mis en ligne le 01 janvier 2013, consulté le 14 novembre 2019. URL : http://journals.openedition.org/ocim/655 ; DOI : 10.4000/ocim.655 


\section{Le développement durable dans la construction d'un Centre de Conservation et $d$ 'Étude}

Séverine Aubert *

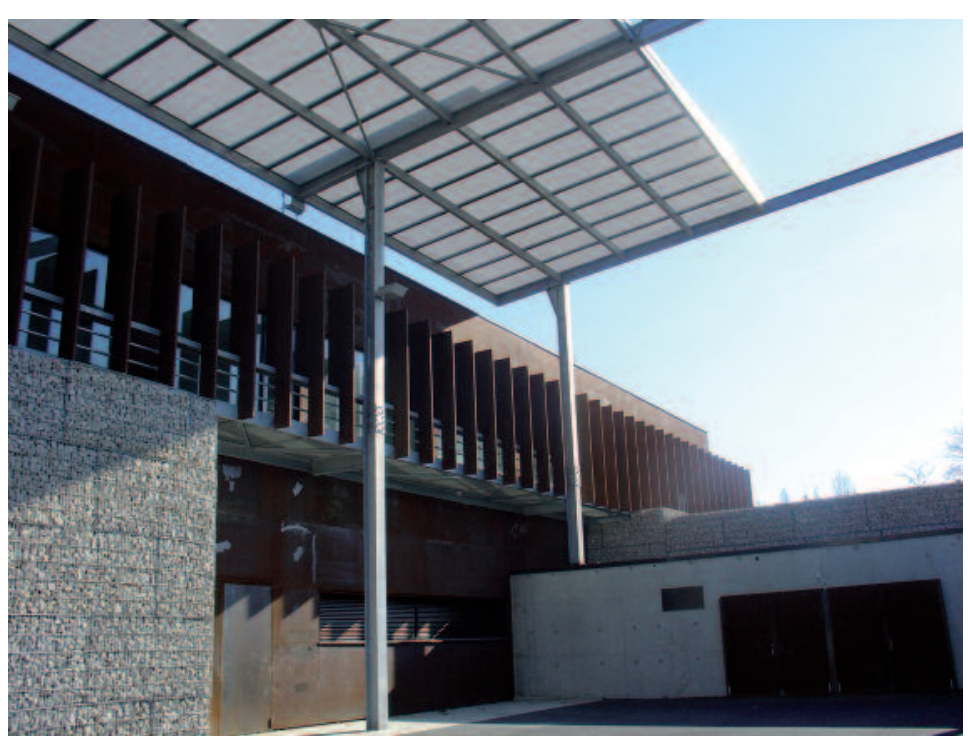

Le Centre de Conservation et d'Étude de Lons-Le-Saunier et ses trois matériaux : gabions en pierre, acier et auvent en polycarbonate ○) S. Aubert

* Séverine Aubert est diplômée du master de Muséologie de l'École du Louvre severine.aubert@yahoo.fr
L'installation du Centre de Conservation et d'Étude de Lons-Le-Saunier, en décembre 2009, a été l'occasion d'une prise en compte simultanée des objectifs de conservation préventive des œuvres et de développement durable dans un projet muséal : elle a permis de considérer différemment la définition d'un programme architectural, tant dans la construction d'un bâtiment que dans son fonctionnement.

Lors de la mise en place du projet d'un nouveau Centre de Conservation et d'Étude comprenant à la fois un lieu de réserves d'œuvres et un lieu d'étude, la Ville de Lons-Le-Saunier, dans le Jura, a eu à conduire un type de réflexion pluridisciplinaire et intégrative. L'analyse de cette démarche permet de mettre en évidence, de préciser et de rapprocher des objectifs propres aux domaines de la technique muséale aux exigences du développement durable ${ }^{(1)}$.

D’un côté, la déontologie des musées donne une grande importance à l'héritage laissé aux générations futures en ce qui concerne les œuvres d'art ou les objets historiques. D'un autre côté, le développement durable est une préoccupation de plus en plus prégnante dans les musées où il conduit progressivement à la définition d'un nouveau référentiel de conception comme d'exploitation. Face aux nouveaux questionnements de la société en matière de protection de l'environnement, le musée a un rôle à jouer.

Une définition du développement durable a été donnée en 1987 par la Commission mondiale sur l'environnement et le développement dans le rapport 
Brundtland : "Un développement qui répond aux besoins des générations du présent sans compromettre la capacité des générations futures à répondre aux leurs » (2). Si ce concept originel de développement durable cherchait à concilier le développement économique avec les préoccupations d'ordre social et la protection des ressources de l'environnement, il s'est ensuite affiné pour devenir une "amélioration des conditions d'existence des communautés humaines, tout en restant dans les limites de la capacité de charge des écosystèmes » (3).

Le musée doit, dans ses nouveaux modèles ${ }^{(4)}$, outre éduquer le public à l'environnement, intégrer le développement durable dans son organisation, que ce soit au niveau économique, social ou écologique pour être considéré comme un nouveau "modèle d'action et d'organisation » (5). Cependant, si la relation entre politique muséale et développement durable n'est pas entièrement nouvelle, elle ne s'est développée que progressivement et de façon sectorielle vers la fin du XXe siècle.

\section{Histoire du rapport entre le développement durable et les musées}

Les musées ont développé au fil du temps de nouvelles compétences et de nouvelles sources de réflexion pour se poser en adéquation avec les questionnements de la société sur le développement durable. Au début du XXe siècle, les musées d'Histoire naturelle traitent, dans leurs discours, de l'environnement. Cependant, ces discours se limitent essentiellement à décrire des spécimens, des groupes, des habitats du milieu naturel. Seules les avancées en matière de paléontologie permettent d'aborder les transformations de l'environnement en décrivant les périodes géologiques.

À partir des années 1960, la question du rôle social du musée est posée. Le musée, comme lieu d'éducation et de pédagogie, renseigne et informe le public, notamment sur les questions d'écologie et d'environnement. Les musées d'Histoire naturelle deviennent alors de véritables centres de recherches sur les modifications de l'environnement. Ils collectent des informations sur la diversité biologique et sur la disparition d'écosystèmes.

En 1992, le centre d'information muséologique UNESCO-ICOM fait paraître une liste d'ouvrages sur le thème du musée et de l'environnement (6) dont la grande majorité concerne les expositions ou les argumentaires développés par les musées pour sensibiliser le public à l'environnement. Sont ainsi présentés les publications des musées d'Histoire naturelle, des jardins botaniques, mais également des écrits sur la biodiversité, la pollution... Notons cependant que quelques titres font état des incidences des pratiques muséales sur l'environnement.

Avec la montée de la pensée environnementale suite aux catastrophes écologiques des années 1990, la société est de plus en plus préoccupée par l'adaptation, à terme, d'objectifs environnementaux, sociaux et économiques. "Le véritable enjeu du musée n'est plus uniquement une mission de conservation, une mise en valeur de ses collections, ni même le succès public, il est lié à une inscription significative et durable de la vie culturelle et sociale des villes, des pays et de la communauté internationale » (7).

Mais si en 1998, lors du Sommet des musées des Amériques à San-José, la notion de développement durable a officiellement intégré le monde muséal, il reste encore difficile de constater une véritable implication des musées pour ces pratiques.

Depuis quelques années, c'est aux États-Unis qu'une véritable conscience des problématiques environnementales s'est faite jour, au-delà de l'intéressement à la réduction des coûts de fonctionnement des institutions. Les établissements muséaux sont appelés à revoir leur politique organisationnelle pour réduire leur impact écologique. Au-delà des initiatives isolées de certains musées, les grandes institutions, édictant la déontologie des musées, s'intéressent au développement durable. Ainsi, l'AAM (American Association of Museums) créé des référentiels pour les musées intégrant le développement durable dans leurs pratiques.

En France, certaines institutions commencent à envisager le développement durable dans leur organisation, mais également lors de constructions de nouveaux bâtiments par respect des recommandations et lois issues du Grenelle de l'Environnement mais également pour bénéficier des avantages financiers liés à la construction durable.

\section{Le développement durable et la conservation préventive}

Le parallèle entre conservation préventive et développement durable est très fort. Selon la définition donnée par le C2RMF ${ }^{(8)}$, la conservation préventive «intervient sur l'ensemble des domaines qui peuvent avoir des incidences et des effets sur l'intégrité d'une collection, d'un objet ou d'une oeuvre d'art, et menacer à terme son existence » (9). Cette dimension de « durabilité » appliquée aux œuvres elles-mêmes 
insiste sur la pérennité et la transmission aux générations futures. La vision holistique du développement durable, prenant en compte les besoins planétaires, rejoint celle de la conservation préventive, se focalisant sur l'ensemble des collections et de leur environnement plus que sur le seul objet.

Deux raisons principales justifient l'importance du développement durable dans un protocole de conservation préventive.

La première est qu'un environnement extérieur plus sain et plus stable (réduisant le risque de catastrophes naturelles) ne peut que contribuer à la conservation préventive des oeuvres conservées dans les musées et les sites historiques.

La deuxième raison concerne le coût du contrôle climatique au sein des musées. Installer et maintenir un contrôle du climat au sein d'un espace coûte à la fois en argent et en énergie, dépenses qui peuvent être évitées par un contrôle passif du climat. Cependant, si les préoccupations financières peuvent être, au départ, un moteur pour intégrer le développement durable dans les musées, les considérations environnementales finissent par gagner les équipes des musées et les pratiques nouvelles se multiplient au sein des établissements.

Si aujourd'hui les objectifs du développement durable paraissent, pour encore de nombreux acteurs du milieu muséal, très éloignés de leurs préoccupations, certains projets cherchent à concilier les besoins de la conservation des œuvres avec des approches environnementales ; c'est le cas du Centre de Conservation et d'Étude de Lons-Le-Saunier.

\section{Le Centre de Conservation et d'Étude de Lons-Le-Saunier}

Le Centre de Conservation et d'Étude de Lons-LeSaunier (CCE) est destiné à abriter les objets de deux musées de la ville : le musée des Beaux-Arts et le musée d'Archéologie. Suite au constat unanime des mauvaises conditions de conservation des œuvres, la Ville a souhaité, d'une part, redéployer les collections en un musée unique, et d'autre part, bâtir un nouvel espace de réserves en périphérie de la ville, afin de remédier aux problèmes de conservation. Ce nouvel espace de réserves représente un exemple abouti d'adéquation entre développement durable et conservation des œuvres.

Les réserves ont été conçues par les architectes Gilles Reichardt et Gilles Ferreux dont l'agence est installée à Lons-Le-Saunier. Le bâtiment, situé en périphérie de la ville, est composé de deux étages

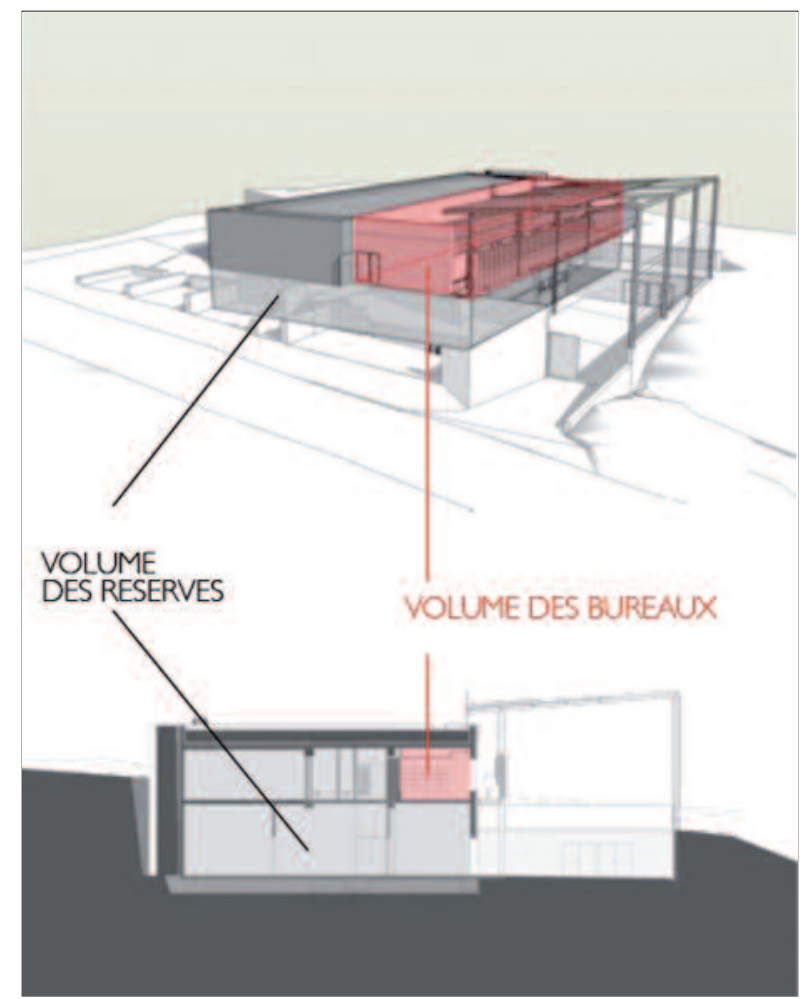

Élévation et coupe du Centre de Conservation et d'Étude, volume des bureaux et des réserves (๑) Gilles Reichardt et Gilles Ferreux Architectes

suivant la déclivité du terrain : le rez-de-cour en sous-sol et le rez-de-chaussée au niveau de la rue. Le rez-de-cour abrite la collection de peintures, de sculptures et de gros objets.

À ces réserves s'ajoutent des espaces de service : un sas, un espace de manutention, une salle de quarantaine, une pièce d'étude et de traitement et un espace de réception. Le rez-de-chaussée abrite les collections organiques, les collections de métaux, les collections traitées et le dépôt du matériel de fouilles. Comme au rez-de-cour, des espaces de service prennent place, cette fois-ci destinés aux chercheurs : cinq bureaux, une bibliothèque, un vestiaire et des sanitaires. La sécurité contre le vol des œuvres est assurée par un système d'alarme antiintrusion. De plus, l'accès pour les chercheurs venant étudier à la bibliothèque est différencié de l'accès aux collections. La déclivité du terrain permet en effet deux accès différents depuis l'extérieur. L'accès aux collections s'effectue depuis l'aire de débarquement, située en rez-de-cour et protégée de la pluie par un auvent. Le rez-de-chaussée au niveau de la rue, permet un accès aux espaces publics et aux espaces de réserves. L'accès aux réserves par cet étage est donc différencié de l'espace public par un système de badge électronique. Les locaux techniques 
sont situés uniquement à l'extérieur du bâtiment, afin d'éviter une entrée de personnel étranger au musée au sein du CCE.

À Lons-Le-Saunier, en plus des exigences liées habituellement à la conservation préventive, le programme du concours architectural impliquait un volet de Haute Qualité Environnementale (10).

Comment ce concept de développement durable, amenant de nouvelles dynamiques et de nouvelles contraintes, a-t-il été intégré aux besoins de conservation préventive inhérents à une réserve de musée ? Le premier défi, celui des économies d'énergie, conduit à fixer la commande d'un bâtiment passif en énergie, c'est-à-dire dont le fonctionnement usuel n'appelle pas un apport d'énergie supplémentaire à celle produite par le bâtiment lui-même.

Le bâtiment se présente comme un pavé semi-enterré recouvert de gabions laissés visibles en façade. La partie enterrée abrite les réserves tandis que la partie émergente, ouverte à la lumière, abrite les bureaux des chercheurs et un centre de documentation. Cette organisation volumétrique du projet cherche à optimiser les échanges énergétiques avec l'environnement extérieur. En effet, la stabilité climatique est mise en place grâce à la dérive lente des températures en milieu tellurique et une très forte isolation réalisée en extérieur afin d'éviter les ponts thermiques au niveau des planchers. L'isolation extérieure est constituée de mousse de verre cellulaire, sur l'ensemble des parties enterrées et de panneaux de fibres de bois pour les parties émergentes.

Cette stabilité thermique est attendue, à la fois au titre du développement durable, mais également au titre de la conservation préventive. La compacité du bâtiment, sa forte isolation, ses façades aveugles et l'utilisation de l'énergie tellurique permettent une très forte inertie thermique. Les dérives de températures extérieures, freinées par les épais murs de béton, les gabions et la couche d'isolation retardent les transferts thermiques à l'intérieur des réserves. À Lons-LeSaunier, l'objectif recherché pour les objets est d'abord une grande stabilité du climat intérieur et des variations très lentes évitant tout choc thermique plutôt qu'un seul niveau de température «idéale ».

De plus, des dispositifs passifs en énergie ont été mis en place afin de renforcer le contrôle de l'environnement des œuvres. Une ventilation double flux avec récupérateur de chaleur, une pompe à chaleur et un puits canadien ont été installés. Ces systèmes peu consommateurs en énergie permettent une ventilation naturelle et un contrôle de la température intérieure. Seules les deux réserves d'objets fragiles

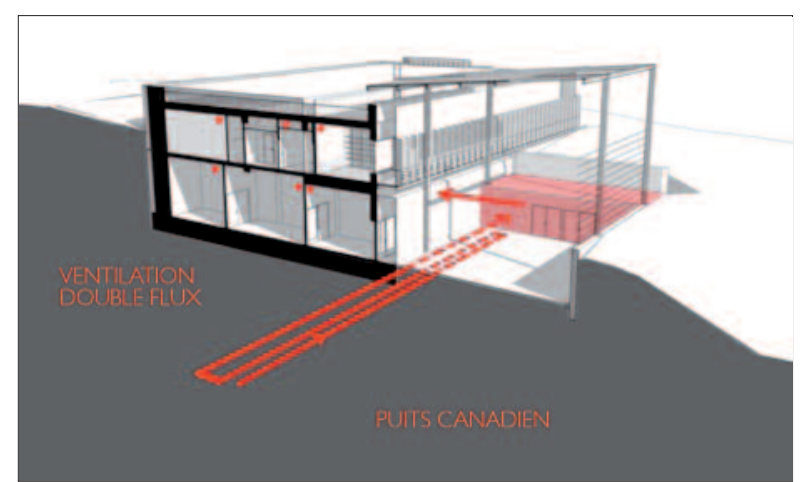

Le dispositif de ventilation double flux (c) Gilles Reichardt et Gilles Ferreux Architectes

seront climatisées en été afin d'assurer un contrôle de la température. Mais l'énergie nécessaire au fonctionnement de ces dispositifs sera fournie par $300 \mathrm{~m}^{2}$ de panneaux photovoltaïques placés sur le toit du bâtiment. Avec une inertie thermique très forte, des moyens de régulation du climat peu consommateurs en énergie et la pose de panneaux photovoltaïques sur le toit, le bâtiment devient alors positif en énergie.

Le deuxième défi, celui du bilan écologique global ou " empreinte écologique », est poursuivi par le choix des matériaux de construction. Si peu de matériaux peuvent être considérés comme parfaits, l'arbitrage s'opère autour d'un juste équilibre entre durabilité, non toxicité, recyclage, renouvelabilité, fabrication peu polluante.

À Lons-Le-Saunier, la structure extérieure du bâtiment est formée de béton armé répondant aux contraintes de durabilité. L'isolation est réalisée avec des panneaux en mousse de verre cellulaire résistante à l'humidité et au feu. Cependant, sa production est polluante et le collage des panneaux sur le bâtiment se fait avec du bitume toxique. Pour une réserve de musée, les possibilités d'utilisation de matériaux isolants sont limitées. En effet, les isolants à base de végétaux ou d'origine animale sont à exclure en raison de leur inflammabilité et de leur attraction sur les insectes.

Afin de réduire le bilan écologique, les ouvriers ont été formés afin de créer une véritable politique de recyclage sur le chantier. Ils ont également bénéficié de formations pour la pose des fenêtres et de l'isolation en verre cellulaire avec deux objectifs principaux : avertir les ouvriers du caractère toxique du bitume pour les inciter à se protéger par le port de masques, et les former à établir une étanchéité complète de l'enveloppe du bâtiment. 
(8) C2RMF : Centre de Recherche et de Restauration des Musées de France

(9) Vademecum de la conservation préventive, 2006. www.culture.gouv.fr/ culture/dmf/vademecum-conservation2006.pdf (consulté le 19 mars 2009 , p. 4 .

(10) La démarche Haute Qualité Envitonnementale se développe en 14 cibles qui se focalisent sur l'éco-construction, l'éco-gestion, le confort et la santé. Pour être certifié HQE par l'AFNOR, une réalisation architecturale doit appliquer des mesures visant à la réalisation de 3 de ces cibles à un haut niveau de performance et à 7 cibles à un niveau de base. De nombreuses collectivités intègrent une démarche HQE à leur programme architectural mais sans en demander la certification par l'AFNOR par la suite. C'est le cas de la ville de Lons-Le-Saunier. De plus, l'Ordre National des Architectes a quitté l'association HQE en 2005, considérant leur démarche comme réductrice.

\section{Bibliographie}

Brophy, S. et Wylie, E. The Green museum, a primer on environmental practice. Royaume-Uni : Altamira Press, 2008, 200 p.

Gaudenzi, V. et Røelens-Duchamp, C. La qualité environnementale dans la production d'expositions, la Lettre de l'OCIM, n99, 2005, pp. 14-20.

Porcedda, A. Musées et développement durable, Les muséums nature de Montréal. Paris : L'Harmattan, 2009, 279 p.

ADEME (Agence de l'Environnement et de la Maîtrise de l'Energie), Qualité environnementale des bâtiments, Manuel à l'usage de la maîtrise d'ouvrage et des acteurs du bâtiment. France : ADEME, juillet 2007.

Giroux, P. Regards en transition : le développement durable et l'exposition muséale, Musées, n²7, 2008, pp. 48-55. 